\title{
Original
}

\section{Patologías relacionadas con las condiciones ambientales de un hospital terciario de la Comunidad de Madrid}

\section{Diseases caused by environmental conditions in a tertiary hospital of Madrid}

\section{Elena Martínez Fernández ', María Fuentes Piñeiro ', Laura Ruiz Ruiz², Jenry Ricardo Borda Olivas ', Margarita Dietl Sagües ${ }^{1}, M{ }^{a}{ }^{a}$ Luisa Rodríguez de la Pinta ${ }^{1}$}

1. Servicio de Prevención de Riesgos Laborales. Hospital Universitario Puerta de Hierro Majadahonda. Madrid. España.

2. Servicio de Prevención Propio del Consejo de Administración del Patrimonio Nacional.

Recibido: 18-11-13

Aceptado: 04-03-14

\section{Correspondencia:}

Elena Martínez Fernández.

Servicio de Prevención de Riesgos Laborales.

Hospital Universitario Puerta de Hierro Majadahonda.

Calle Joaquín Rodrigo N. ${ }^{\circ} 2$

28220 Majadahonda. Madrid. España

Tel: 91 1916417. Fax: 911917653

E-mail: elenamarfer@hotmail.com

Resumen

Introducción: El Síndrome del edificio enfermo (SEE) cursa con una mayor incidencia de síntomas como cefaleas, irritación de mucosas y sensación de fatiga, que mejoran o incluso desaparecen al abandonar el edificio.

La Organización Mundial de la Salud (OMS) estima que este problema afecta al 30\% de los edificios modernos y causa molestias al 10-30\% de sus ocupantes.

Objetivo: Determinar si los síntomas manifestados por los trabajadores de un hospital terciario de la Comunidad de Madrid se corresponden con los síntomas que definen el SEE.

Material y Método: Estudio descriptivo transversal. Se pasó un cuestionario a un grupo de trabajadores, seleccionados de forma aleatoria y voluntaria.

Resultados: Se recogieron 207 cuestionarios, 164 (79,2\%) de mujeres y 43 (20,8\%) de hombres. La edad media fue 41,4 años, 42,6 en mujeres y 36,6 en hombres ( $\mathrm{p}<0,01)$. Se encontró reagrupamiento de casos en algunas áreas del hospital (laboratorios y quirófanos) y se midieron condiciones ambientales: humedad 23-26\% (valores recomendados superiores al 30\%) y temperatura normal.

Se encontraron 92 casos $(44,4 \%)$ con síntomas que definen el SEE.

Conclusiones: El edificio en el que se desarrolla nuestro estudio cumple los criterios que se establecen en la definición de SEE.

Med Segur Trab (Internet) 2014; 60 (234) 53-63

Palabras claves: Síndrome edificio enfermo, condiciones ambientales, síntomas inespecíficos.

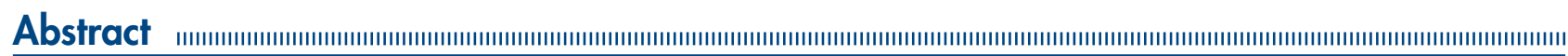

Introduction: Sick Building Syndrome (SBS) has more incidence of headache, irritation of mucous tissues and fatigue among workers. These symptoms get better or even disappear when workers leave the building. 
objectives: The main aim of our research project was to check if the symptoms that some workers of one Tertiary Hospital in the Community of Madrid showed were those of SBS.

Material and Method: For that purpose, we carried out a "cross-sectional descriptive study" through a questionnaire completed by staff members of the hospital both randomly selected and willing to take part.

Results: 207 questionnaires were collected, 164 (79.2\%) of women and 43 (20.8\%) of men. The average age was 41.4 years, 42.6 in women and 36.6 in men $(\mathrm{p}<0,01)$. More cases were found in certain areas of the hospital (laboratories and operating theaters). We were measured environmental conditions were measured: humidity 23 to $26 \%$ (recommended values above 30\%) and normal temperature. 92 cases (44.4\%) were found with symptoms that define the ESS (> $20 \%$ of those studied).

Conclusions: According to the given results, we can establish that the analysed building meets all the requirements set by the definition of the SBS.

Med Segur Trab (Internet) 2014; 60 (234) 53-63

Keywords: Sick Building Syndrome, environmental conditions, nonspecific symptoms. 


\section{INTRODUCCIÓN}

Cada día adquieren mayor importancia los efectos que puede provocar la mala calidad de aire en la salud de los trabajadores. Estos se han visto potenciados por el diseño y construcción de edificios cada vez más herméticos (oficinas, inmuebles públicos...) en los que la recirculación del aire adquiere un papel relevante con objeto de asegurar un ahorro de energía. Sin embargo, los ambientes que no disponen de ventilación natural pueden ser áreas de exposición a contaminantes ${ }^{(1)}$.

El término "calidad de aire interior" se aplica a ambientes de interiores no industriales: edificios de oficinas, edificios públicos, viviendas particulares... ${ }^{(2)}$. En los últimos años ha cobrado especial relevancia al asociarse al término "síndrome del edificio enfermo", que comprende un amplio rango de síntomas o enfermedades que las personas que trabajan o habitan en dicho edificio atribuyen al edificio en sí. Cuidando la calidad del aire o ambiente interior, se cuida de la salud de las personas que viven o trabajan, en definitiva, que pasan un tiempo considerable, en el interior de dicho edificio.

La Estrategia española de calidad del aire también hace referencia a la calidad del aire interior y lo menciona especialmente dentro del grupo de trabajo de Calidad de aire y salud ${ }^{(3)}$

La incidencia real del problema es desconocida, pero la OMS estima que afecta al 30\% de los edificios modernos y que causa molestias al 10-30\% de sus ocupantes. Los síntomas que han sido comunicados en diferentes estudios sobre el tema y que conforman el síndrome son principalmente: irritación de ojos, nariz y garganta; sequedad de piel y mucosas; eritema cutáneo; fatiga mental, somnolencia; cefaleas, vértigo; mayor incidencia de infecciones de vías respiratorias altas; dificultad respiratoria, jadeo, roncus, sibilancias, cuadros asma-like; disfonía, tos; alteraciones del gusto y del olfato y náuseas ${ }^{(4)}$.

Estos síntomas no suelen ser severos y, al no ocasionar un exceso de bajas por enfermedad, se tiende a menudo a minimizar su importancia; sin embargo se traducen en una sensación general de disconfort. Cuando llegan a afectar a más del veinte por ciento de los ocupantes de un edificio, se habla del SEE ${ }^{(1,5)}$.

Esta sintomatología se comporta de forma característica, aumentando a lo largo de la jornada laboral y remitiendo o mejorando al abandonar el lugar de trabajo, desapareciendo incluso durante las vacaciones.

Los factores más comúnmente citados como responsables del síndrome son:

Agentes químicos (formaldehído, compuestos orgánicos volátiles, polvo, fibras, dióxido de carbono, monóxido de carbono, óxidos de nitrógeno, ozono...); Agentes biológicos (bacterias, hongos, esporas, toxinas, ácaros...); Agentes físicos (iluminación, ruido, vibraciones, ambiente térmico, humedad relativa, ventilación...); Agentes psicosociales (organización del trabajo, relaciones interpersonales, etc.).

Respecto a los agentes físicos, el Real Decreto 486/1997 de 14 de abril establece las disposiciones mínimas de seguridad y salud en los lugares de trabajo ${ }^{(6,7)}$ (ver anexo 4 ).

Estudios realizados en Europa, sugieren que el SEE está asociado con los sistemas mecánicos de ventilación que utilizan humidificadores y refrigerantes ${ }^{(8)}$. La percepción de los síntomas relacionados con el trabajo también depende de la categoría laboral y el sexo. El origen de una deficiente calidad del aire interior puede ser diferente para distintos edificios, pudiendo ser en ocasiones el propio sistema de ventilación.

Existen dificultades para definir lo que se entiende por Edificio Enfermo y por SEE ${ }^{(9)}$. En general, los edificios enfermos suelen presentar problemas relacionados con las siguientes características: están equipados con aire acondicionado, aunque también pueden estar ventilados de forma natural; sus ocupantes presentan quejas referentes a su salud en una proporción mayor a la que sería razonable esperar ( $>20 \%$ ); las causas son difíciles de identificar, dado que en muchos casos tienen un origen multifactorial. El SEE 
es el nombre que se da al conjunto de síntomas diversos que presentan, predominantemente, los individuos en estos edificios y que, en general, no van acompañados de ninguna lesión orgánica o signo físico, diagnosticándose, a menudo, por exclusión.

La OMS diferencia entre dos tipos distintos de edificio enfermo:

- Los edificios temporalmente enfermos, entre los que se incluyen edificios nuevos o de reciente remodelación, en los que los síntomas disminuyen y desaparecen con el tiempo, aproximadamente medio año.

- Los edificios permanentemente enfermos, cuando los síntomas persisten, a menudo durante años, a pesar de haberse tomado medidas para solucionar los problemas $^{(10)}$.

Las razones que justifican este estudio son:

Desde la apertura de este hospital, se observó que un número importante de trabajadores acudían al Servicio de Prevención de Riesgos Laborales con quejas relacionadas con síntomas similares a los que conformaban el SEE. Por ello se planteó que, una vez descartadas otras causas (infecciones o antecedentes personales), pudiesen existir causas relacionadas con el propio edificio (ambientales) que justificasen esta sintomatología.

Es un edificio de reciente construcción, está equipado con un sistema de climatización forzada de aire, hermético (las ventanas no se pueden abrir) y existe relación temporal positiva entre la ocupación del edificio y la aparición de los síntomas, etc.

\section{OBJETIVOS}

Objetivo principal: Determinar si los síntomas manifestados por los trabajadores de un hospital terciario de la Comunidad de Madrid se correspondían con los síntomas que definen el SEE.

Objetivos secundarios:

1. Determinar la sintomatología de los trabajadores de dicho hospital según categoría profesional, turno de trabajo, sexo, edad, puesto de trabajo.

2. Conocer las características ambientales de diferentes zonas del hospital e identificar posibles factores de riesgo.

3. Valorar si existía reagrupación de casos en determinadas áreas hospitalarias.

4. Determinar si la sintomatología ya estaba presente en los trabajadores antes del traslado.

5. Establecer una posible relación entre los hallazgos encontrados y el SEE.

\section{MATERIAL Y MÉTODO}

Diseño: Se realizó un estudio descriptivo transversal durante los meses de septiembre de 2011 a diciembre de 2012.

Se llevó a cabo mediante la entrega de un cuestionario a los trabajadores implicados (n=207), basado en la Nota Técnica de Prevención $380^{(11)}$, la Metodología de evaluación de el Síndrome del Edificio Enfermo del INSHT ${ }^{(12)}$ y el libro Calidad de Aire Interior ${ }^{(13)}$.

El cuestionario contenía preguntas específicas de respuesta corta y cerrada. Se incluyó una pregunta abierta donde se reagruparon dos tipos de síntomas que no estaba en los cuestionarios base aunque si aparece en la bibliografía consultada.

Se explicó a los trabajadores participantes en qué consistía el estudio mediante el envío de una carta y/o reunión informativa. Se clasificaron las diferentes áreas donde se realizaron las encuestas siguiendo el RD 486/1997 de Lugares de Trabajo ${ }^{(14)}$, la 
norma UNE 100713 sobre instalaciones de acondicionamiento de aire en los hospitales (15), y la norma americana de la ASHRAE (American Society of Heathing, Refrigerating and Air-Conditioning Engineers) ${ }^{(16)}$ en:

- Urgencias.

- Consultas.

- Hospitalización.

- Laboratorios.

- Quirófanos y Unidad de Cuidados Intensivos (UCI).

- Radiología.

- Servicios generales: lencería, cocina, admisión, recursos humanos, etc.

Cada área se subdividió en zonas, según la distribución física del hospital.

Se llevaban cinco encuestas a cada zona, una vez entregadas y recogidas las cumplimentadas independientemente de que no se cumplimentasen todas, no se volvía a pasar por allí. Se repartieron en total 350 encuestas.

Población: La población a estudio fue la plantilla de trabajadores del Servicio Madrileño de Salud de un hospital terciario de la Comunidad de Madrid.

Los criterios de inclusión fueron: trabajadores del Hospital Puerta de Hierro que desarrollen la mayor parte de su jornada laboral (al menos un $80 \%$ ) dentro del edificio y que acudan de manera habitual a su puesto de trabajo. No se incluye a trabajadores que sólo van de forma esporádica, p.e., para hacer guardias 1-2 veces al mes, cubrir bajas temporales de pocos días de duración, becarios, alumnos que hacen prácticas unas horas, etc.

Los criterios de exclusión fueron:

- Trabajadores diagnosticados de enfermedades alérgicas, oculares, respiratorias, etc., que presentaran síntomas similares a los descritos por el SEE, lo cual podría crear confusión en cuanto al origen de los síntomas.

- Trabajadores que presentaran este tipo de síntomas con anterioridad a la ocupación del edificio.

- Personas que trabajaban a tiempo parcial.

El tamaño muestral fue calculado en base al número de trabajadores del hospital 3750 aproximadamente.

\section{Variables}

- Expuesto: Se consideró expuesto al trabajador que pasaba rutinariamente la mayor parte de su jornada laboral (al menos el 80\%) dentro de las instalaciones del Hospital Terciario seleccionado a estudio.

- Efecto: Se consideró efecto a la aparición, aumento y persistencia en el lugar de trabajo y durante varias semanas de al menos tres de los siguientes síntomas (de uno o varios grupos):

- Síntomas oculares: escozor y/o enrojecimiento, lagrimeo.

- Síntomas de vías respiratorias superiores: rinorrea, congestión nasal, picor nasal, hemorragia nasal, estornudos en salva, sequedad de garganta, dolor de garganta, ronquera.

- Síntomas generales: dolor de cabeza, somnolencia, letargo, dificultad para concentrarse, irritabilidad, náuseas, mareos.

- Síntomas pulmonares: opresión torácica, sensación de ahogo, pitidos, tos seca.

- Síntomas cutáneos: eritema, sequedad cutánea, prurito generalizado, prurito localizado (estos dos últimos grupos de síntomas fueron reagrupados en el cuestionario como otros"). 
- Caso: se consideró caso todo trabajador que presentaba el efecto, siempre y cuando se diese la circunstancia de que los síntomas apareciesen en el lugar de trabajo y desapareciesen o mejorasen al abandonar el edificio (al final de la jornada laboral, días de descanso o en periodo vacacional). No se consideró caso a trabajadores diagnosticados de enfermedades alérgicas, oculares, respiratorias, etc., que presentasen síntomas similares a los descritos en el SEE antes del inicio del estudio.

Otras variables: sexo (hombre, mujer), edad en años, turno (mañana: 8-15h, tarde; 15-22h, noche: 22-8h; otros), categoría profesional (médicos, enfermeras, auxiliares de enfermería, etc.), tiempo trabajado en el hospital y en el puesto de trabajo en meses, permanencia en el edificio en horas.

Recogida de datos: Se recogieron datos a través de un cuestionario específico. Al existir reagrupamiento de casos en determinadas zonas como laboratorios, se realizaron mediciones puntuales de los parámetros ambientales que se estimó convenientes (temperatura, humedad, etc.), tanto en esas zonas como en las que no había concentración de casos, con el fin de comparar resultados. Para ello se utilizaron un termohigrómetro, un termonanemómetro y el equipo Monitor Gas Probe IAQ de BW Technologies.

Análisis de datos: Se realizó un análisis descriptivo de las variables relacionadas con la sintomatología del SEE. Se realizaron cruces básicos con variables de interés como Servicio, Categoría profesional, Turnicidad, etc. Test de Chi-cuadrado para comparar proporciones y test de $\mathrm{t}$ de Student o análisis de varianza para comparar medias. En todos estos casos se asumió un error a del $5 \%$.

Estos datos fueron introducidos y analizados mediante el programa informático SPSS 15.0 (Statistical Package for Social Sciences).

\section{Limitaciones del Estudio}

- Sesgo de selección: la predisposición a responder al cuestionario podía ser debida a la condición de "afectado" o "enfermo", con lo que aumentaría de forma artificial la prevalencia de los síntomas, de ahí la importancia de hacer una selección aleatoria de los sujetos, teniendo prevista la sustitución en caso de no respuesta.

- Sesgo de memoria: cuando se preguntó a los trabajadores por antecedentes de exposición o por si habían padecido esos síntomas con anterioridad a la ocupación del edificio, existía la posibilidad de olvido.

- Sesgo voluntario: ya que todos los datos que fueron recogidos en la encuesta específica provenían de trabajadores que participaron por iniciativa propia y los que no lo hicieron podían tener una opinión diferente que no pudimos analizar.

\section{Cumplimiento de Normas Éticas y Legales}

Se trabajó por el bienestar de la población que participó en nuestro estudio, según los principios de beneficencia, justicia y no maleficencia y según la Ley de protección de datos 15/1999.

El método de estudio garantizó el bienestar de cada participante, ya que la recogida de las encuestas fue anónima y voluntaria. Todos los datos que fueron recogidos para su posterior análisis mediante base de datos informatizada, cumplían el "deber de secreto" de todo estudio científico.

\section{RESULTADOS}

Se recogieron un total de 207 cuestionarios (tabla 1), de los cuales 164 fueron mujeres $(79,2 \%)$ y 43 hombres $(20,8 \%)$. En cuanto a la categoría profesional, $27,1 \%$ correspondieron a médicos, 24,6\% a enfermeros, 16,9\% a auxiliares de enfermería, 15,9\% a administrativos y $12,6 \%$ a técnicos de laboratorio. La mayoría eran de turno mañana $(78,7 \%)$ y el resto de turno tarde $(21,3 \%)$. 
La edad media de la población en estudio fue de 41,4, siendo la edad media en mujeres (42,6 años) mayor que la media de edad en hombres (37 años) en 5,6 años $(\mathrm{p}<0,001)$.

En cuanto a la media de tiempo trabajado, la mayor parte de los trabajadores estaba desde la apertura del centro (36,8 de los 44 meses que lleva en funcionamiento); trabajando en el mismo servicio una media de 34,2 meses, y la media de horas por jornada laboral se situó en las 7,5 horas diarias.

Se encontró un total de 92 trabajadores con síntomas que cumplían con la definición de caso de SEE (44,4\%), de los cuales 82 eran mujeres $(89,1 \%)$ y 10 eran hombres $(10,9 \%)$, siendo significativa esta diferencia $(p<0,01)$. La edad de los casos de SEE era de 42.2.

De los trabajadores que no fueron considerados caso, 49 tenían síntomas compatibles con SEE previos a la ocupación del nuevo edificio, que empeoraban desde que trabajan en él y mejoraban al abandonar el mismo. La edad media de estos trabajadores era 40.8. Estos trabajadores no fueron clasificados como casos de SEE para no crear confusión en el estudio.

Tabla 1. Características de la Población

\begin{tabular}{|c|c|c|}
\hline & Frecuencia & $\%$ \\
\hline Total & 207 & \\
\hline Sexo & 207 & \\
\hline Hombres & 43 & 20,8 \\
\hline Mujeres & 164 & 79,2 \\
\hline \multicolumn{3}{|l|}{ Categoría Profesional } \\
\hline Médico & 56 & 27,1 \\
\hline Enfermera/o & 51 & 24,6 \\
\hline Auxiliar de Enfermería & 35 & 16,9 \\
\hline Auxiliar Administrativo & 33 & 15,9 \\
\hline Técnico de Laboratorio & 26 & 12,6 \\
\hline Otro & 6 & 2,9 \\
\hline \multicolumn{3}{|l|}{ Turno } \\
\hline Mañana & 163 & 78,7 \\
\hline Tarde & 44 & 21,3 \\
\hline Noche & 0 & 0 \\
\hline \multicolumn{3}{|l|}{ Áreas Hospitalarias } \\
\hline Consultas & 85 & 41,1 \\
\hline Laboratorios & 33 & 15,9 \\
\hline Hospitalización & 25 & 12,1 \\
\hline Urgencias & 21 & 10,1 \\
\hline Quirófanos y UCI & 19 & 9,2 \\
\hline Servicios Generales & 19 & 9,2 \\
\hline Radiología & 5 & 2,4 \\
\hline
\end{tabular}

En cuanto a la categoría profesional (tabla 2), de los 92 casos de SEE encontrados, 22 eran enfermeras (27,3\%), 20 eran auxiliares de enfermería (22,7\%) y 18 eran médicos $(21,6 \%)$.

En relación a los turnos de trabajo, en el de mañana se encontraron 69 casos de SEE de los 163 encuestados, y en el turno de tarde 23 casos de los 44 encuestados, no habiendo diferencias estadísticamente significativas. 
Tabla 2. Casos de SEE según Categoría Profesional

\begin{tabular}{lcc}
\hline & Casos & $\%$ \\
\hline Categoría Profesional & & \\
Médico & 18 & 19,6 \\
Enfermera/o & 22 & 23,9 \\
Auxiliar de Enfermería & 20 & 21,7 \\
Auxiliar Administrativo & 13 & 14,1 \\
Técnico de Laboratorio & 17 & 18,5 \\
Otro & 2 & 2,2 \\
\hline
\end{tabular}

El área del hospital donde se encontraron más casos (tabla 3) fueron los laboratorios, con 24 casos $(72,8 \%$ de los trabajadores de ese área), seguido de la zona de consultas con 24 casos (28,2\% de trabajadores de consultas) y Quirófanos - UCI con 15 casos $(78,9 \%$ de los trabajadores de esa zona).

Tabla 3. Casos de SEE según áreas hospitalarias

\begin{tabular}{lcc}
\hline & Casos & $\%$ \\
\hline Áreas Hospitalarias & & \\
Consultas & 24 & 26,1 \\
Laboratorios & 24 & 26,1 \\
Hospitalización & 11 & 11,9 \\
Urgencias & 9 & 9,8 \\
Quirófanos y UCI & 15 & 16,3 \\
Servicios Generales & 8 & 8,7 \\
Radiología & 1 & 1,1 \\
\hline
\end{tabular}

El tiempo medio de trabajo en el centro relacionado con los casos de SEE, se situó en los 38,7meses, mientras que en los no casos de SEE fue de 35,2 meses, siendo esta diferencia estadísticamente significativa $(\mathrm{P}=0,041)$. En cuanto al tiempo trabajado en el mismo servicio y su relación con los casos de SEE, se situó en 36,6 meses mientras que en los no casos fue de 32,3 meses, siendo esta diferencia también estadísticamente significativa $(\mathrm{P}=0,028)$. El tiempo medio en horas de la jornada laboral de los casos y no casos de SEE fue de 7,58 h frente a 7,5 h, no existiendo diferencias significativas entre ambos.

Se realizaron mediciones higiénicas de duración variable en aquellos lugares donde existía reagrupación de casos. El equipo de medición se ubicó aproximadamente en el centro de cada uno de los recintos obteniéndose valores de humedad relativa similares en todas las zonas entre un 23 y un $26 \%$. Se hicieron también mediciones de temperatura, encontrándose en todos los casos valores entre 24 y $26^{\circ} \mathrm{C}$. En cuanto a la concentración de $\mathrm{CO} 2$, los valores obtenidos se encontraban en un rango entre 550-600 ppm. El mismo día se realizaron mediciones en una de las zonas donde no existía concentración de casos (Hospitalización), obteniéndose valores de humedad relativa entre 33-34\%, temperatura entre 24,2 y $25,2^{\circ} \mathrm{C}$, y valores de CO2 entre 648 y 675 ppm de CO2. También se midió en el exterior del hospital y los valores registrados fueron una humedad relativa del $34 \%$, una temperatura de $15^{\circ} \mathrm{C}$ y la concentración de CO2 de 466ppm.

\section{DISCUSIÓN}

Debido a la escasez de trabajos publicados sobre este tema no se han podido contrastar nuestros resultados.

Se encontró que más del 20\% del personal, en este estudio el 44,4\%, padecía los síntomas que definían el SEE. Los trabajadores no presentaban estos síntomas antes de la ocupación del edificio. 
Los síntomas empeoraban a lo largo de la jornada laboral y mejoraban o desaparecían al finalizar la misma, en días de descanso y en periodo vacacional.

Se observó reagrupamiento de casos en determinadas zonas del hospital (quirófanos y laboratorios).

Una cuestión de interés era saber si el sexo influía a la hora de padecer o no el SEE, ya que las mujeres presentaban más casos que los hombres, en concreto de los 92 casos, 82 eran mujeres y 10 eran hombres. Un 50\% de mujeres se vio afectado frente a un $23,8 \%$ de hombres, siendo esta diferencia estadísticamente significativa.

Si además de la edad tenemos en cuenta el sexo se observo que la media de edad en mujeres que presentaban el SEE era similar a las que no presentaban SEE; con una $\mathrm{p}=0,78$; en hombres la media de edad de los hombres que presentaban síntomas compatibles con SEE es semejante a los que no lo presentaban, con una p=0,19. En ninguno de los dos casos la diferencia era estadísticamente significativa.

Se observó que influía el tiempo trabajado en el hospital a la hora de padecer o no SEE, con una media de 39 meses en los casos de SEE, mientras que en los no casos fue de 35 meses, siendo esta diferencia significativa $(\mathrm{p}=0,041)$. Se observó que cuanto más tiempo permanecían los trabajadores en un edificio de estas características, más síntomas podían presentar.

Respecto a las áreas del hospital, las zonas de concentración de casos fueron la zona de laboratorios, donde un $72,7 \%$ de los encuestados padecían SEE y quirófanos - UCI con un $78,9 \%$ de trabajadores afectados. Se midieron en estas zonas humedad relativa, temperatura y valores de $\mathrm{CO} 2$, encontrándose valores de humedad relativa entre un $23 \%$ y un $26 \%$, frente a los valores establecidos en el RD 486/1997 (humedad relativa entre el 30 y 70\%) y la norma UNE 100713:2005 (humedad relativa del 45\%) ${ }^{(15)}$, siendo probable que dichos reagrupamientos fuesen debidos a esos valores tan bajos de humedad.

\section{CONCLUSIONES}

1. En el presente estudio se observó que se cumplían los criterios que definen el SEE, es decir, los ocupantes del edificio presentan quejas referentes a su salud en una proporción mayor a la que sería razonable esperar (>20\%)

2. Los síntomas provocadas por el SEE son inespecíficos y de difícil diagnóstico: Con frecuencia son atribuidos a otros factores o patologías. Es importante reconocer los síntomas descritos por los trabajadores de edificios de estas características y una vez descartadas otras causas pensar en la posible relación con las condiciones ambientales de su lugar de trabajo para poder adoptar las medidas pertinentes.

3. La Ley 31/1995, de Prevención de Riesgos Laborales, establece la obligatoriedad de investigar todos los riesgos para la salud y seguridad de los trabajadores. Las condiciones ambientales en los lugares de trabajo no deben constituir una fuente de riesgo, incomodidad o molestia para los trabajadores. Se deben mejorar las condiciones ambientales en los lugares de trabajo para evitar el disconfort de los trabajadores y el absentismo laboral (en este caso aumentado un 10\% los valores de humedad relativa).

4. Se debe concienciar a los responsables de las empresas de la necesidad de invertir tiempo y dinero en solucionar este tipo de problemas, pues con un bajo gasto se obtienen grandes beneficios mejorando las condiciones de trabajo y disminuyendo el absentismo. 


\section{AGRADECIMIENTOS}

La elaboración de este artículo no ha contado con apoyos financieros.

Los autores de este artículo no tienen conflictos financieros.

Trabajo realizado dentro del Programa Científico de Formación MIR de Medicina del Trabajo desarrollado por la Escuela Nacional de Medicina del Trabajo del Instituto de Salud Carlos II en convenio con las Unidad Docente de Medicina del Trabajo de Madrid.

\section{REFERENCIAS BIBLIOGRÁFICAS}

1. Peña Castiñeira M. Cómo detectar y tratar el síndrome del edificio enfermo.Gestión Práctica de Riesgos Laborales. 2006; 28:33.

2. Junta de Andalucía. Plan Andaluz de salud ambiental. 2008-2012. Consejería de Salud, Consejería de Medio Ambiente.

3. Ministerio de Medio ambiente. Estrategia española de calidad del aire. Ministerio de Medio Ambiente. 2005.

4. Instituto Nacional de Seguridad e Higiene en el Trabajo NTP 290: El síndrome del edificio enfermo: cuestionario para su detección. Colección Notas Técnicas de Prevención. M. ${ }^{\circ}$ de Empleo y Seguridad Social. INSHT Madrid. [acceso 22 Agosto 2011].

http://www.insht.es/Inshtweb/Contenidos/Documentacion/FichasTecnicas/NTP/Ficheros/201a300/ ntp_290.pdf

5. Instituto Nacional de Seguridad e Higiene en el Trabajo NTP 243: Ambientes cerrados: calidad del aire. Colección Notas Técnicas de Prevención. M. ${ }^{\circ}$ de Empleo y Seguridad Social. INSHT Madrid. [acceso 22 Agosto 2011].

http://www.insht.es/Inshtweb/contenidos/Documentacion/FichasTecnicas/NTP/Ficheros/201a300/ ntp_243.pdf

6. Instituto Nacional de Seguridad e Higiene en el Trabajo NTP 779: Bienestar térmico. Criterios de diseño para ambientes térmicos confortables. Colección Notas Técnicas de Prevención. M. ${ }^{\circ}$ de Empleo y Seguridad Social. INSHT Madrid. [acceso 22 Agosto 2011].

http://www.insht.es/Inshtweb/contenidos/Documentacion/FichasTecnicas/NTP/Ficheros/752a783/ntp_779.pdf

7. Disposiciones Mínimas de Seguridad y Salud en los lugares de trabajo. Real Decreto 486/1997 del 14 de Abril. Boletín Oficial del Estado, n. ${ }^{\circ}$ 7, (23/04/1997).

8. K Reijula, C Sundman-Digert. Assessment of indoor air problems at work with a questionnaire. Occup environ Med. 2004; 61:33-38

9. Instituto Nacional de Seguridad e Higiene en el Trabajo NTP 289: Síndrome del edificio enfermo: factores de riesgo. Colección Notas Técnicas de Prevención. M. ${ }^{\circ}$ de Empleo y Seguridad Social. INSHT Madrid.

10. WORLD HEALTH ORGANIZATION (WHO) Indoor air quality research EURO Reports and Studies No 103, WHO Regional Office for Europe. Copenhagen 1986.

11. Instituto Nacional de Seguridad e Higiene en el Trabajo NTP 380: El síndrome del edificio enfermo: cuestionario simplificado. Colección Notas Técnicas de Prevención. M. ${ }^{\circ}$ de Empleo y Seguridad Social. INSHT Madrid. [acceso 22 Agosto 2011].

http://www.insht.es/Inshtweb/contenidos/Documentacion/FichasTecnicas/NTP/Ficheros/301a400/ntp_380.pdf

12. Berenguer Subils M. ${ }^{a}$ J, Guardino Solá X, Hernández Calleja A, Martí Solé M. ${ }^{a}$ C, Nogareda Cuixart C, Solé Gómez M. ${ }^{a}$ D. El Síndrome del Edificio Enfermo: guía práctica para su evaluación. Madrid: Instituto Nacional de Seguridad e Higiene en el Trabajo; 2004.

13. Bartual Sánchez, Berenguer Subils M. ${ }^{a}$ J, Bernal Domínguez F. Calidad de Aire Interior. Madrid: Instituto Nacional de Seguridad e Higiene en el Trabajo; 2001.

14. RD 486/1997 de Lugares de Trabajo.

15. Instalaciones de acondicionamiento de aire en hospitales. UNE 100713.2005. Reglamento de Instalaciones Térmicas en los Edificios (RITE). 2. ${ }^{a}$ ed, (07/09/2005).

16. ASHRAE Standard 62-1989 Ventilation for Acceptable Indoor Air Quailty American Society of Heating, Refrigerating, and Air-Conditioning Engineers, Inc., Atlanta, GA. 1989). 


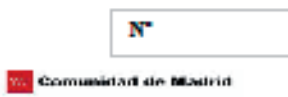

\section{CUJESTIONARTO SOBRE LA CALIDAD DEILAIRE EN ESPACIOS INTERTORES}

Se está llevando a cabo un estudio para tratar de mejorar las Condiciones de Trabajo en el edificio en el que usted desarrolla su actividad laboral Para ello necesitamos que responda a este cuestionario. No es necesario que se identifique, ni que firme, ya que es totalmente anónimo.

Gracias por su colaboración.

Fecha:

1. CATEGORIA PROFESIONAL (médico, DUE, auxiliar, celador ...):

2. SERVICIO:

3. TURNO HABITUAL: Mañana $\square \quad$ Tarde $\square \quad$ Noche $\square$ Otros

4. $\mathrm{EDAD}$ SEXO: Hombre $\square \quad$ Mujer $\square$

5. ¿Cuánto tiempo hace que trabaja en el HUPH Majadahonda? Años: Meses:

6. ¿Cuánto tiempo hace que trabaja en el mismo servicio? Años: Meses:

7. Horas de permanencia en el edificio (jornada laboral habitual):

8. ¿Trabaja más de 4 horas al dia con ordenadores?: $\mathrm{Si}$ No

9. ¿Padece algún diagnóstico previo al inicio de su actividad laboral en este nuevo hospital?:

$\begin{array}{llllll}\text { CONJUNTIVITIS: } & \text { Si } & \text { No } & \text { DERMATITIS: } & \text { Si } & \text { No } \\ \text { RDNITIS: } & \text { Si } & \text { No } & \text { CEFALEAMIGRANTA: } & \text { Si } & \text { No } \\ \text { SINUSITIS: } & \text { Si } & \text { No } & \text { OJO SECO } & \text { Si } & \text { No }\end{array}$

OTROS (Especificar)

Importante: Por favor, anotar tan sólo aquellos sintomas o molestias que le hayan ocurrido en los últimos treinta días en su puesto de trabajo y que mejoren al abandonar el edificio donde trabaja (ya sea inmediatamente o después de algunas horas).

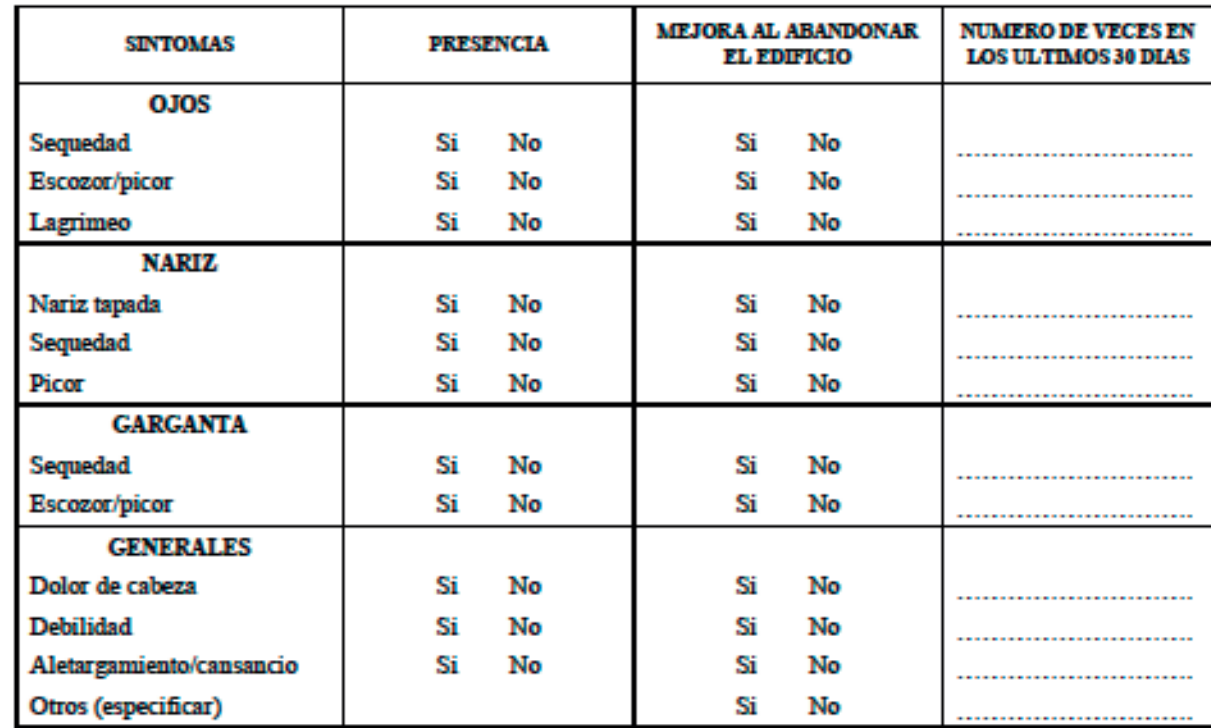

